\title{
Characterization and interpretation of the Edge Snake in between Type-I ELMs at ASDEX Upgrade
}

\author{
F Sommer $^{1}$, S Günter ${ }^{1}$, A Kallenbach ${ }^{1}$, M Maraschek ${ }^{1}$, \\ J Boom ${ }^{1}$, R Fischer ${ }^{1}$, N Hicks ${ }^{1}$, N C Luhmann Jr ${ }^{2}$, HK Park ${ }^{3}$, \\ B Reiter ${ }^{1}$, R Wenninger ${ }^{4}, \mathrm{E}_{\text {Wolfrum }}{ }^{1}$ and the ASDEX Upgrade Team \\ ${ }^{1}$ Max-Planck-Institut für Plasmaphysik, Boltzmannstr. 2, D-85748 Garching, \\ EURATOM Association, Germany \\ ${ }^{2}$ University of California at Davis, Davis, CA 95616 USA \\ ${ }^{3}$ POSTECH, Pahang, Gyeongbuk, 790-784, Korea \\ ${ }^{4}$ Universitätssternwarte der Ludwig-Maximilians-Universität, D-81679 München, Germany \\ E-mail: fabian.sommer@ipp.mpg.de
}

\begin{abstract}
A new magneto-hydro-dynamic instability called 'Edge Snake', which was found in 2006 at the Tokamak ASDEX Upgrade during type-I ELMy H-modes, is investigated. It is located within the separatrix in the region of high temperature and density gradients and has a toroidal mode number of $n=1$. The Edge Snake consists of a radially and poloidally strongly localized current wire, in which the temperature and density profiles flatten. This significant reduction of pressure gradient leads to a reduction of the neoclassical Bootstrap current and can plausibly explain the drive of the instability. The experimental observations point towards a magnetic island with a defect current inside the O-point of the island. The Edge Snake is compared to similar instabilities at JET, DIII-D and ASDEX Upgrade.
\end{abstract}

\section{Introduction}

With increasing size of fusion experiments, the peak load of expelled energy and particles during an Edge Localized Mode (ELM) on the plasma facing components increases [1]. For future reactors like ITER such loads are not tolerable. Hence great effort is underway to increase the understanding of the edge transport to mitigate or even avoid ELMs. While studying the various edge parameters, e.g. magnetic field and line integrated plasma radiation a new distinctive pattern was found. Owing to the localization of the underlying instability at the edge and the resemblance of the signals to the already described Core Snakes $[2,3]$ it was called Edge Snake.

In section 2 the Edge Snake is characterized by means of various diagnostics covering a broad range of plasma parameters $\left(\dot{B}_{\mathrm{r}}, \dot{B}_{\mathrm{pol}}, T_{\mathrm{e}}(1 D, 2 D), n_{\mathrm{e}}\right.$, line integrated plasma radiation, $H_{\alpha}$ radiation). In section 3 the implications of the experimental findings will be discussed and possible theoretical explanations of this phenomenon will be proposed. In section 4 the Edge Snake will be compared to similar instabilities at JET, DIII-D and ASDEX Upgrade. 


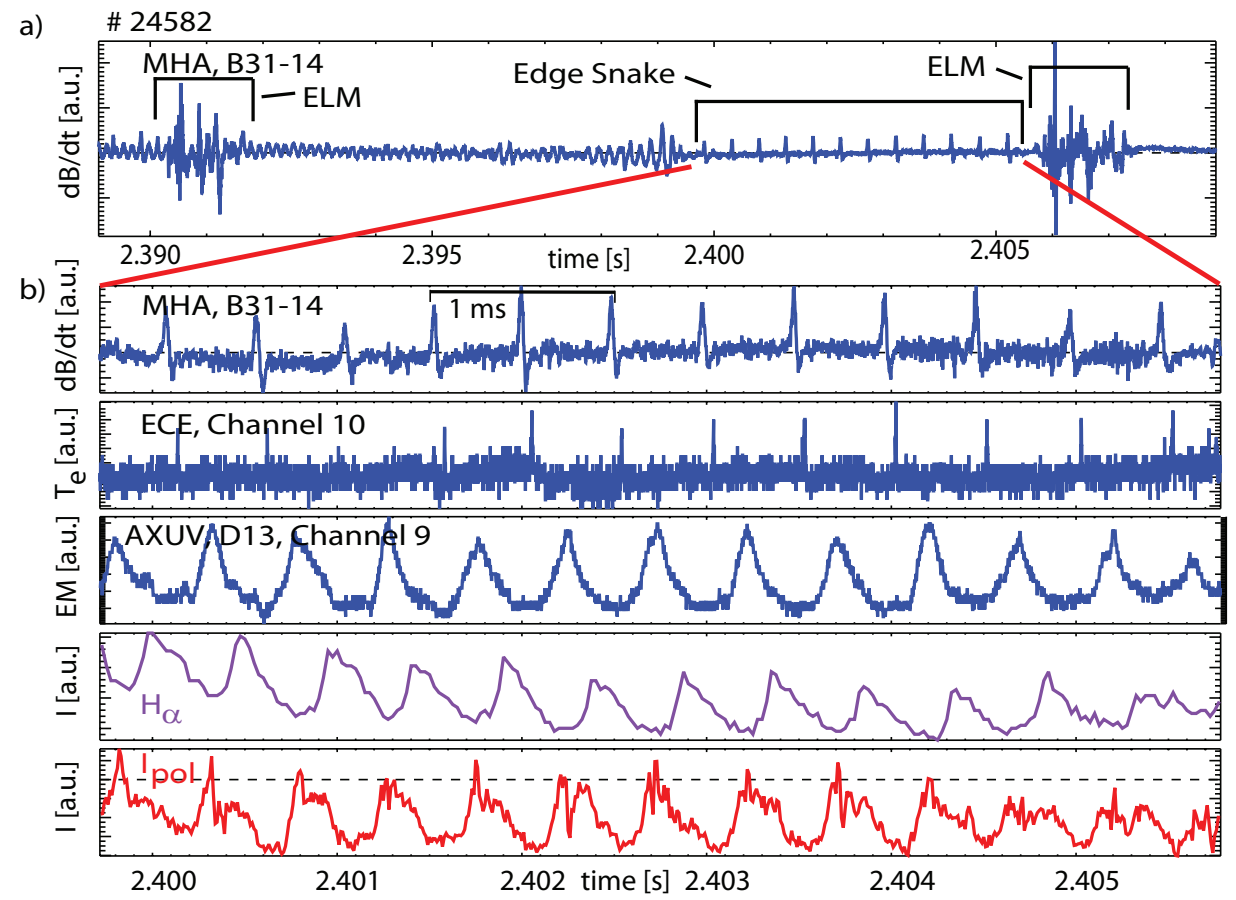

Figure 1. (a) Time trace of the derivative of the radial magnetic field, showing 2 consecutive ELMs and the typical comb-like signal structure of the Edge Snake.

(b) Typical signals of the Edge Snake on various plasma parameters: magnetic field (MHA), electron temperature (ECE), line integrated plasma radiation (AXUV), $\mathrm{H}_{\alpha}$ radiation in the divertor $\left(\mathrm{H}_{\alpha}\right)$ and shunt currents in the divertor tiles $\left(\mathrm{I}_{\mathrm{pol}}\right)$.

\section{Experimental observations}

The magneto-hydro-dynamic phenomenon Edge Snake was analyzed with magnetic probes (so called Ballooning coils), designed to measure the derivative of the radial magnetic field close to the separatrix. A typical signal from these probes can be seen in Figure 1 (a). The distinct inharmonic character of the perturbation of the magnetic field is clearly visible between two consecutive ELMs. The Edge Snake occurs only during high recycling Type-I ELMy $\mathrm{H}$-modes with $q_{95}<6$ and large central line averaged densities of typically $8 \cdot 10^{19} \mathrm{~m}^{-3}$. The usual lifetime of the Edge Snake between two ELMs is around $4 \mathrm{~ms}$ but can extend up to $20 \mathrm{~ms}$. The amplitudes of the different measurements do not show a particular increase or decrease during its occurrence. Typically the appearance of an Edge Snake is not systematically in between many consecutive ELM cycles: sometimes it emerges and disappears somewhere in between two ELMs while on other occasions it begins immediately afterwards or is terminated by an ELM.

In addition to the magnetic signal the Edge Snake can be seen in various other plasma parameters like the electron temperature, measured by the Electron Cyclotron Emission plasma diagnostic (ECE [4]) and the line integrated plasma radiation, measured by semiconductor pinhole cameras and ranging from UV to Soft X-ray (Absolute power measurement in the 


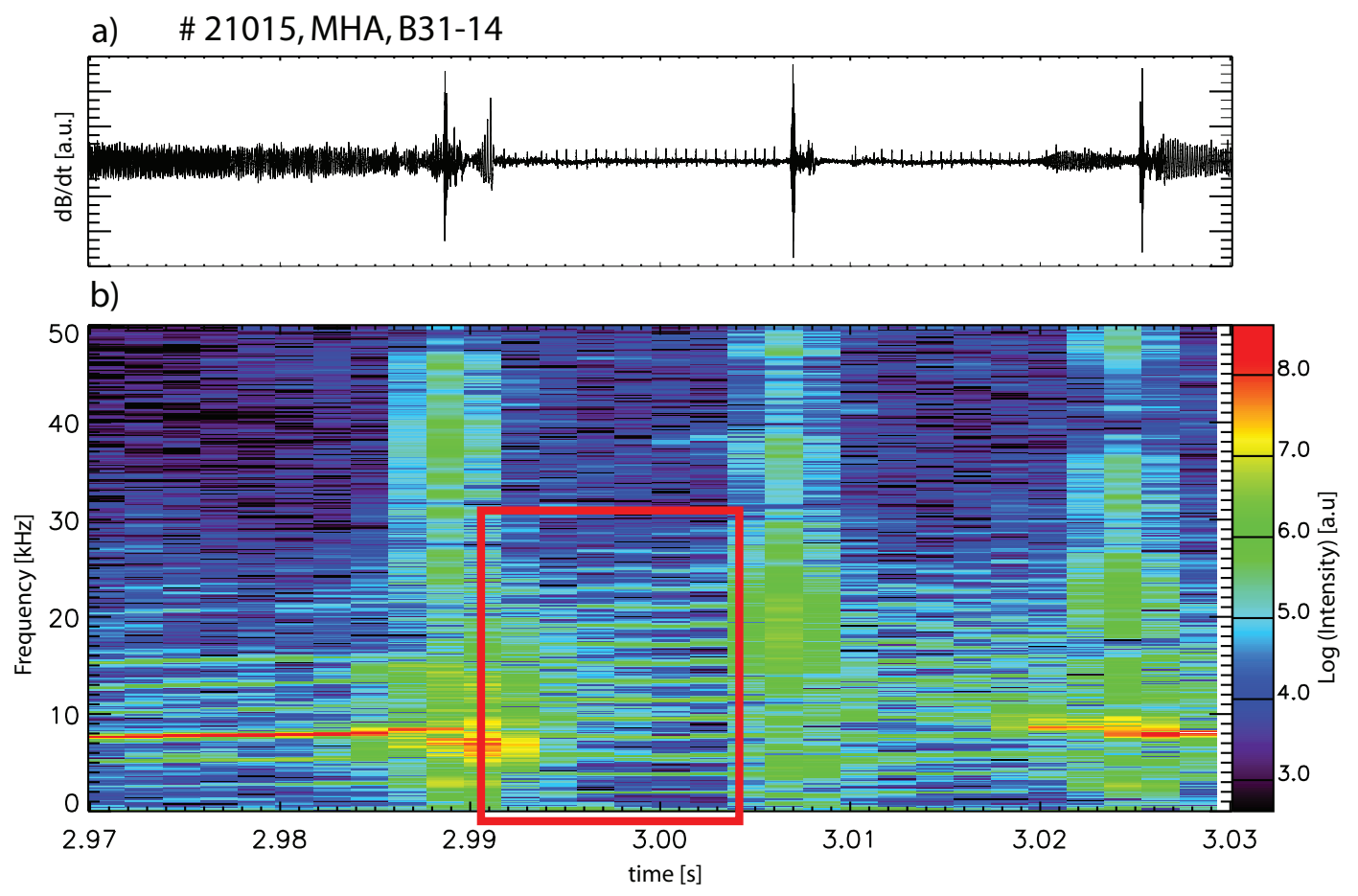

Figure 2. (a) Time trace of the derivative of the radial magnetic field, showing 3 consecutive ELMs and the typical comb-like signal structure of the Edge Snake.

(b) Spectrogram of the magnetic signal, showing up to 15 harmonics.

eXtreme Ultra Violet spectral range, AXUV [5]). Furthermore, the perturbation can be seen in the $\mathrm{H}_{\alpha}$ radiation measured in the lower divertor. The poloidal currents flowing from the plasma onto the divertor, which are measured via shunts in the target plates ( $\mathrm{I}_{\mathrm{pol}}[6]$ ) also show the distinctive pattern of the Edge Snake. Time traces of all these measurements are shown in Figure 1(b), where the inharmonic structure of the signal can be seen in most cases. Some signals are however broadened due to geometrical effects and line transitions of not fully ionized atoms.

\subsection{Frequency and mode numbers derived from magnetic measurements}

From the magnetic signals a fundamental frequency of $2.0 \pm 0.2 \mathrm{kHz}$ can be derived. Having a closer look at the spectrograms up to 15 additional harmonics can be observed, which are caused by the strong inharmonic nature of the signal (Figure 2(b) around 3.00 seconds). The toroidal mode number can be determined with the toroidal Ballooning coil array, which covers half of the circumference of the torus. With only very few exceptions (discussed later) it has a fundamental value of $n=1$ with many harmonics. The analysis of the poloidal Ballooning coil array, which covers only $60 \mathrm{deg}$ on the low field side, leads to a minimal poloidal mode number of $m>3.5$. Since in most cases the lifetime of the Edge Snake was too short to analyze it by means of a Fourier transformation, a method of determining the phase difference between the channels via a correlation analysis was implemented. This method leads to a local poloidal phase velocity around the midplane on the low field side of around $10 \mathrm{krad} / \mathrm{s}$ in 
the electron diamagnetic drift direction. The toroidal phase velocity calculated likewise has a value of around $13 \mathrm{krad} / \mathrm{s}$. Both speeds are only projections of the movement of the helical instability on the two perpendicular directions. By dividing the two velocities and accounting for the different rotation centers the pitch angle of the magnetic field lines can be obtained assuming the alignment of the instability with the latter. The resulting inclination of 0.2 , compared with equilibrium calculations [7], locates the Edge Snake at a $\rho_{\text {pol }}$ between 0.9 and 1.0, which resembles the outermost $5 \mathrm{~cm}$ inside the separatrix.

To deduce the exact poloidal mode number the phase shift of the Mirnov coil array was simulated. These coils span the entire poloidal circumference and measure the derivative of the poloidal magnetic field just inside the vacuum chamber. The higher harmonics of the perturbation are damped in the signals of these coils, because of their larger distance to the plasma and their different orientation. Therefore the phase shift could not be observed all the way around the torus. The simulation was done with the Mirnov Interpretation Code (MIC [8]), in which a sinusoidal perturbation current distribution $j_{\text {pert }}$ with a variable $m$-number and a large exponent $k$ is assumed to account for the constrained current: $j_{\text {pert }} \propto \sin \left(\theta^{*}(\theta) \cdot m\right)^{k}$. Here $\theta^{*}(\theta)$ is the straight field line angle. By comparing the simulation with the experimental measurements the best accordance could be achieved with a fundamental poloidal mode number of $m=6$. This locates the Edge Snake on the $q=6$ surface, which lies typically in the steep gradient zone around $\rho_{\text {pol }} \approx 0.98$.

\subsection{Polarity and magnitude of the current}

The polarity and magnitude of the perturbation current causing the Edge Snake can be determined by integration of the time traces of the Ballooning coils. A schematic of a current wire moving upwards past the coils is shown in Figure 3 (c), where the current in the wire is flowing out of the plane of projection and against the normal ohmic and Bootstrap plasma current. This configuration produces a counter clockwise magnetic field, which is first (position 1) directed against the measuring direction of the coil, when the current wire is approaching it. Thus the coil measures a negative radial magnetic field, which becomes zero when the current wire is located exactly in front of the coil (position 2). The measured field then switches to a positive value while the current wire is moving further upwards (position 3) and decays slowly due to the growing distance between current wire and coil. Exactly the same pattern is shown in the integrated magnetic measurements (Figure 3 (b)), which implies a defect (counter) current inside the Edge Snake.

Due to the strong radial and poloidal localization of the current wire, the flowing current can be calculated with the formula of Biot Savart. For that purpose, an infinitesimal current distribution on the $q=6$ surface was assumed. The distance was taken as the minimal separation between this surface, deduced from equilibrium calculations and one Mirnov coil, which measures the derivative of the poloidal magnetic field, $B_{p o l}$. Integration of this signal leads to Figure 3 (d). The negative deviance of the poloidal magnetic field from the unperturbed case 
a) \#24607, MHA, B31-09
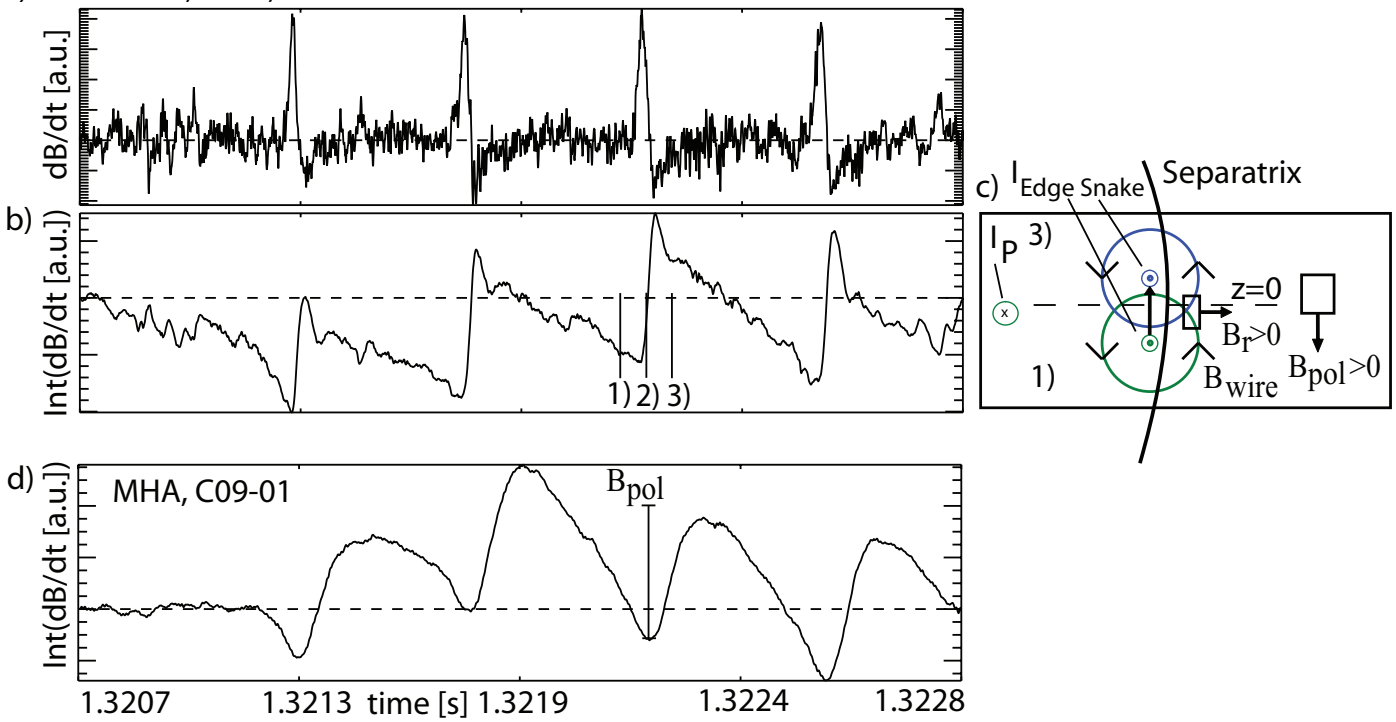

Figure 3. (a) Time trace of the measured derivative of the radial magnetic field.

(b) Radial magnetic field obtained by integration of time trace a).

(c) Schematic of a current wire passing one coil along the field lines.

(d) Poloidal magnetic field obtained by integration of one Mirnov coil.

shows the counter current character of this instability. By using this deviance and taking into account the angle between current wire (pitch angle) and orientation of the coil, the flowing current can be evaluated. In several discharges at multiple time points this value is always in the range of $150 \pm 30$ Ampere.

\subsection{Electron temperature and density behavior}

The distinctive pattern of the Edge Snake can also be seen in the electron temperature on channels just inside the separatrix in the steep gradient zone (Figure 4 (a)). Shown here is one channel, where a temperature drop of about $100 \mathrm{eV}$ is apparent with a background temperature of around $300 \mathrm{eV}$. Because the ECE is a localized and fast measurement, the poloidal extension of the Edge Snake can be calculated. The full width at half maximum of the time, the Edge Snake needs to pass the line of sight of the ECE lies around $10 \mu \mathrm{s}$ (Figure 4 (a)). By multiplying this value with the prior determined poloidal velocity and the radius of the flux surface, the Edge Snake has a poloidal extension of only $5 \mathrm{~cm}$. The ratio of this extent and the poloidal distance of two current wires is 1:50 and shows the extreme poloidal localization of the temperature perturbation. Assuming a strong link between the temperature perturbation and the region of the defect current by the changed Bootstrap current the poloidal localization of the current wire is also evident. The radial structures of the Edge Snake can be seen from the edge profiles of the electron temperature. In Figure 4 (b) the averaged profile (solid black curve), which resembles the period between two passings of the Edge Snake, is compared with profiles taken from single time points at various passings of the Edge Snake through the line of sight of the ECE (dashed colored curves). The shown time points are indicated in 

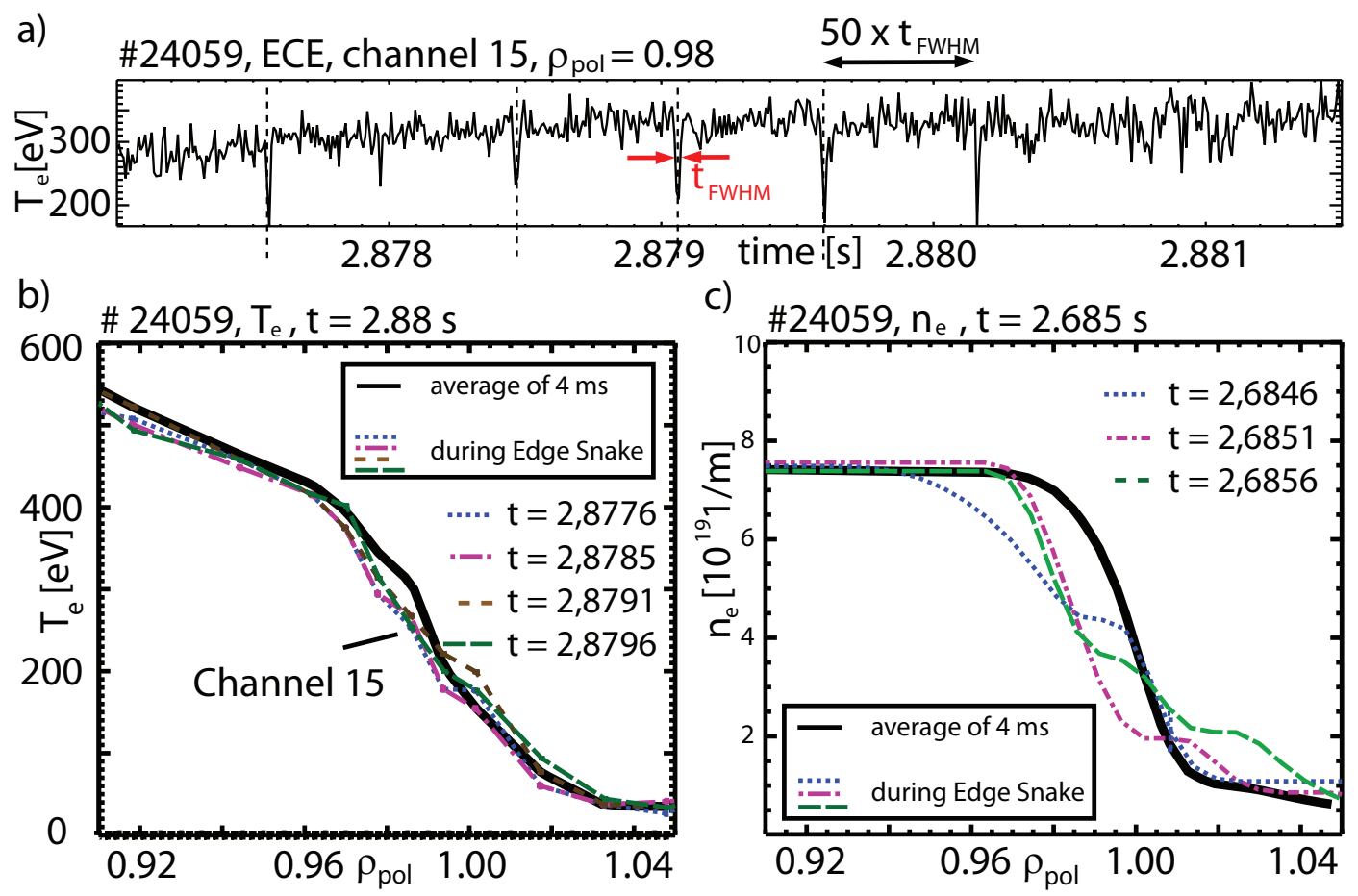

Figure 4. (a) Time trace of one channel of the electron temperature just inside the separatrix. Time points taken for the profile shown as dashed vertical lines.

(b) Edge profile of the electron temperature and electron density (c) showing the flattening in the steep gradient zone due to the Edge Snake.

Figure 4 (a) with dashed vertical lines. The inversion of the profile to the plasma equilibrium can be seen at a radial position of $\rho_{\text {pol }}=0,97$ to 1 , which is consistent with the findings of the simulation performed with the MIC Code. This inversion is not a global phenomenon which occurs on the entire magnetic surface but is very localized to the perturbation of only a few $\mathrm{cm}^{2}$ caused by the Edge Snake.

The edge profiles of the electron density are measured with the Lithium Beam Diagnostic [9]. Its signal shows a flattening of the profiles, whose minimal gradient is at the same radial position as the temperature measurements (Figure 4 (c)). The solid black curve represents the inter Edge Snake averaged profile, whereas the dashed colored curves are measured during short time intervals of consecutive passings of the Edge Snake through the Li-Beam. Due to an $R_{\text {out }}$ scan to optimize the data acquisition, no case is available where both $T_{e}$ and $n_{e}$ are measured at the same time point with the needed accuracy to compare both values directly. But the assumption is nearby, that the passing of the Edge Snake causes a poloidal and toroidal very localized temperature and density flattening. The radial extent of about 1.5 $\mathrm{cm}$ and the poloidal extent of $5 \mathrm{~cm}$ lead to a cross sectional area of about $7 \mathrm{~cm}^{2}$. With the measured current of 150 Ampere in the filament a current density of $0.2 \mathrm{MA} / \mathrm{m}^{2}$ has been calculated. This value is about $20 \%$ of the unperturbed Bootstrap current at the location of the Edge Snake. This current was calculated with the CLISTE Code [10], using the unperturbed electron temperature and density gradient profiles in the steep gradient zone. 


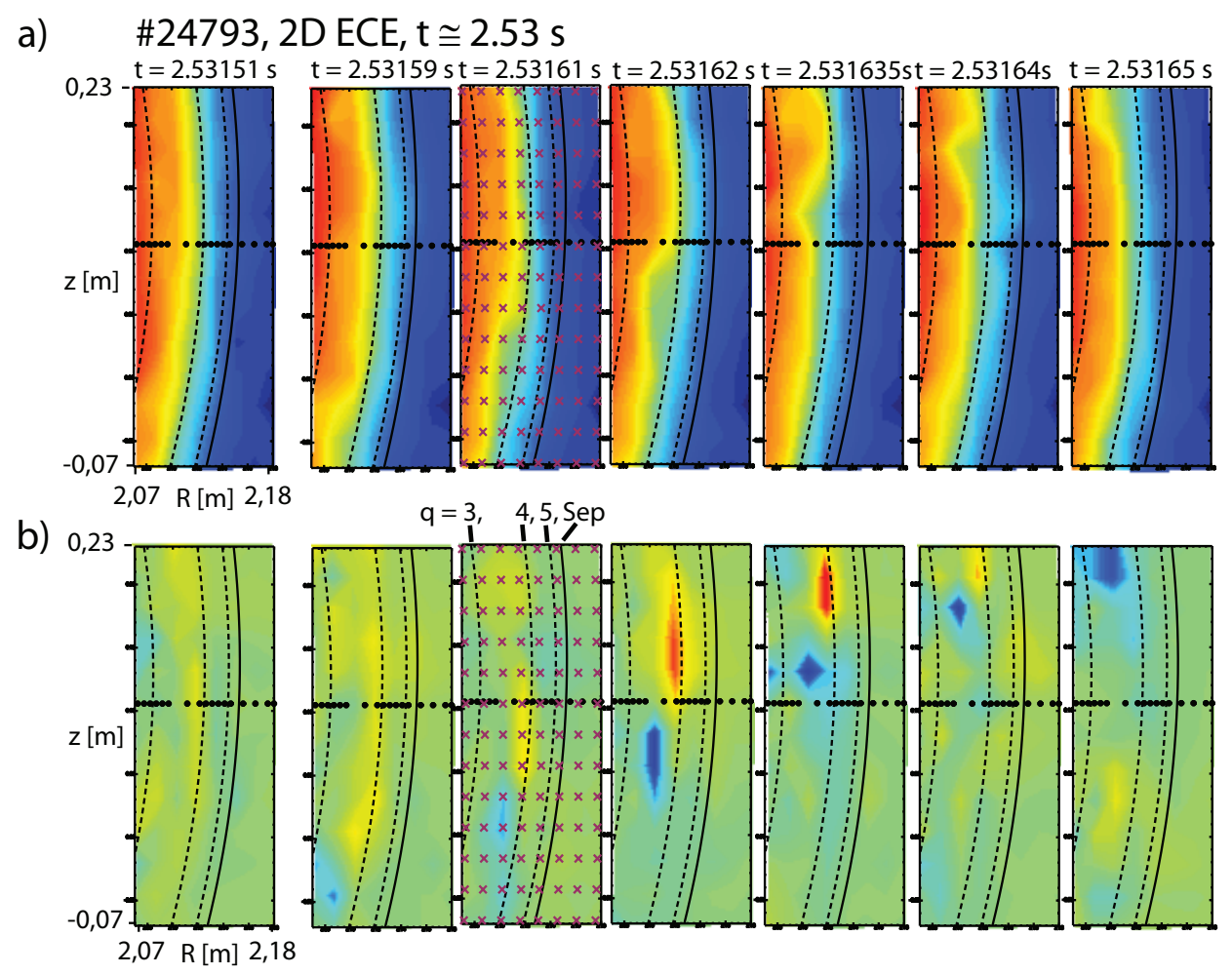

Figure 5. (a) Visualization of the upward movement of the Edge Snake in the 2D electron temperature at consecutive time frames and (b) deviation from the equilibrium electron temperature by subtraction of the average temperature of every channel for the same time frames.

\subsection{Occurrence in the $2 D$ electron temperature measurement}

The Edge Snake is also visible in the 2D electron temperature measured by the 2D ECE [11], which covers a $11 \times 30 \mathrm{~cm}$ cross section of the plasma around the separatrix on the low field side. In the first row of Figure 5 the movement of the Edge Snake for consecutive time frames can be seen, while the first plot shows the unperturbed case. For a better analysis of the data the separatrix and some rational surfaces deduced from equilibrium calculations for the unperturbed case are also shown in the plot. While the position of the separatrix is identified by a temperature value of around $100 \mathrm{eV}$, the radial locations of the q-surfaces have an error bar of around $1 \mathrm{~cm}$ due to their determination by the externally measured magnetic field. This value is in the typical range of the distance between two rational surfaces.

Clearly visible is the upward movement of the instability around the $\mathrm{q}=4 \pm 1$ surface which is consistent with the magnetic measurements within the error bars of the ECE and magnetic measurements. The previously derived poloidal extension of around $5-7 \mathrm{~cm}$ (approximate length of the entire perturbation) and radial extension of around $1.5 \mathrm{~cm}$ (distance between two channels), can be confirmed, keeping in mind the fluctuation of the data. Since the perturbation of the electron temperature can be clearly linked to the perturbation on the magnetics and shows the same inharmonic behavior, a similar ratio between the distance and the poloidal 
extension of two consecutive passings of the Edge Snake can be expected. This ratio again has an approximate value of 50:1.

The plots in the second row also show a representation of the electron temperature at the same points of space and time as the ones in the first row. In contrast to these ones they display the deviation from the equilibrium temperature of every channel. This is deduced by subtracting the average value of the temperature of each channel from the signal. In this representation of the data the variation of the temperature by the Edge Snake is even more pronounced. It consists of a first part, which is either hotter than the unperturbed plasma or in which plasma is pushed outwards into cooler regions. This is followed by a second part, which is cooler than the unperturbed case or in which plasma is pulled inside into hotter areas. Additionally a difference of the radial position of the two parts can be seen, where the hotter first part is located more to the outside than the cooler part. This disposition could already be seen in the $1 \mathrm{D}$ electron temperature profiles and is in accordance with the theory of a magnetic island.

The interpretation of the data in this representation has to be carried out carefully. The perturbation appears as two clearly toroidally and radially separated structures, whereas looking at the original data it seems rather to consist of a bending of the flux surfaces.

\subsection{Radiation}

Looking at the time traces of the AXUV diagnostic a variation of the shape of the signals from discharge to discharge can be seen. This variation depends on the exact temperature and density at the rational surface in the steep gradient zone, where the Edge Snake is located. Two cases are shown in Figure 6 for the same channel but from different discharges. The small phase shift compared to the same magnetic channel originates from the different poloidal and toroidal positions of the two diagnostics. In most cases the signal exists of a strong reduction of the radiation shown in Figure 6 (a). The other signature, only observed in discharges with a higher divertor electron temperature, is shown in Figure 6 (b).

In general it is not possible to ascertain the contribution of bremsstrahlung and line radiation of several impurities, especially in the edge region of the plasma. Here neither the exact temperature, nor the exact density nor the exact impurity concentrations are known. One possible explanation of the first pattern is a reduction of the radiation, mostly bremsstrahlung, due to the reduced density and mostly reduced temperature inside the island. One hypothesis for the sawtooth shaped signals is the inward shift of the density profiles produced by the passing of the Edge Snake. Hence fewer atoms in the line of sight of the diagnostic can contribute to the radiation. A slow recovery phase occurs until the shape of the unperturbed steep gradient zone is reached again. 

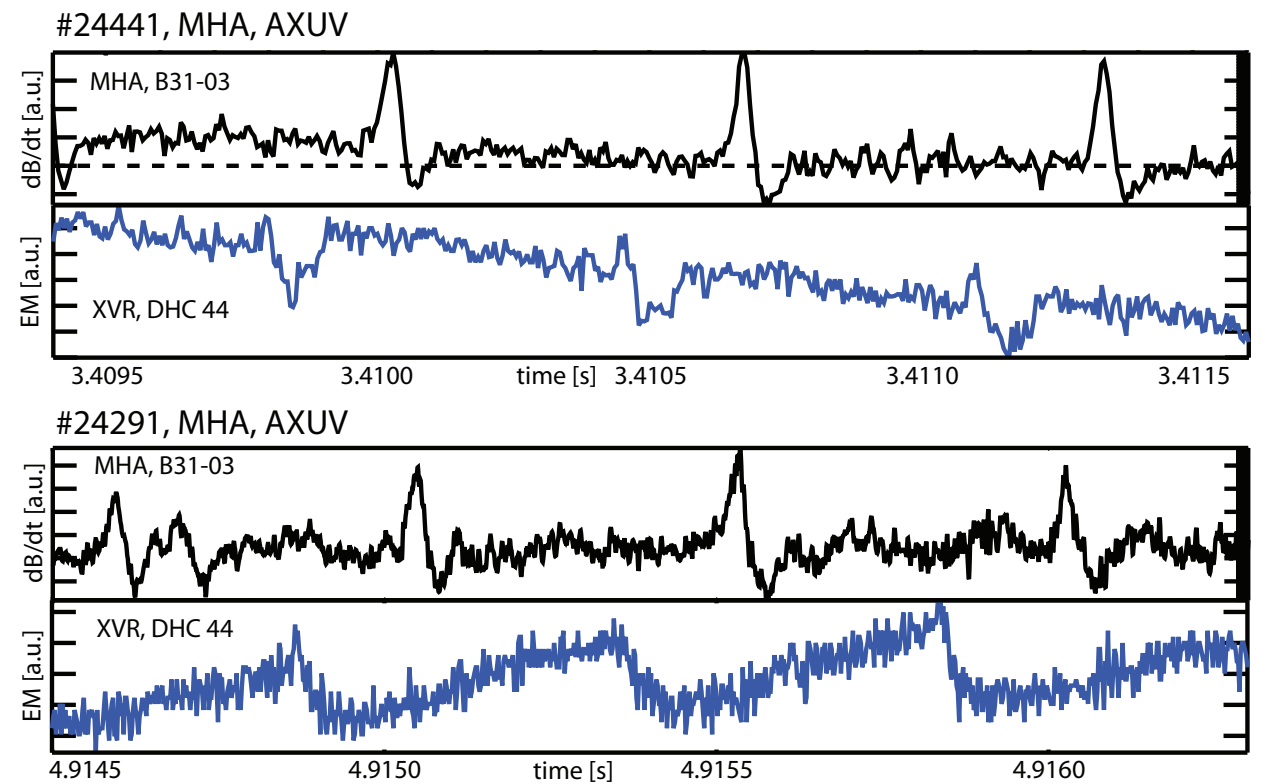

Figure 6. Time traces of the same channel of the line integrated plasma radiation measurement (AXUV) in comparison to one magnetic coil, showing a difference in the shape of the signal, depending on the exact temperature and density in the steep gradient zone.

\subsection{Divertor diagnostics}

The measurement of the poloidal currents which flow from the scrape off layer onto the divertor as well as the $\mathrm{H}_{\alpha}$ signal clearly show the distinctive pattern of the Edge Snake (Figure 1 (b)). Despite its localization inside the separatrix and its small radial and poloidal extension the Edge Snake has a clear influence through the scrape off layer into the divertor region.

\subsection{Exotic cases of the Edge Snake}

Apart from the typical case of an $n=1, m=6$ instability, cases were found with an $n=2, m=12$ structure, which implies two current wires separated toroidally by 180 degree. Additionally even more exotic cases exist, where these two wires are separated toroidally by $\frac{1}{3} \cdot 360 \mathrm{deg}$ (Figure 7). It seems as if an $n=3, m=18$ structure exists on the rational surface, where only two branches are occupied with a current wire and thus are visible on the diagnostics. Furthermore, a few cases were found with an $n=4$ to 6 and $m=6 \cdot n$ structure, where only $n-1$ branches could be seen in the measurements, while one branch did not show any amplitude. This behavior points towards a substructure composed of the higher harmonics of the current distribution. This substructure is mostly suppressed but can arise to a measurable magnitude. 


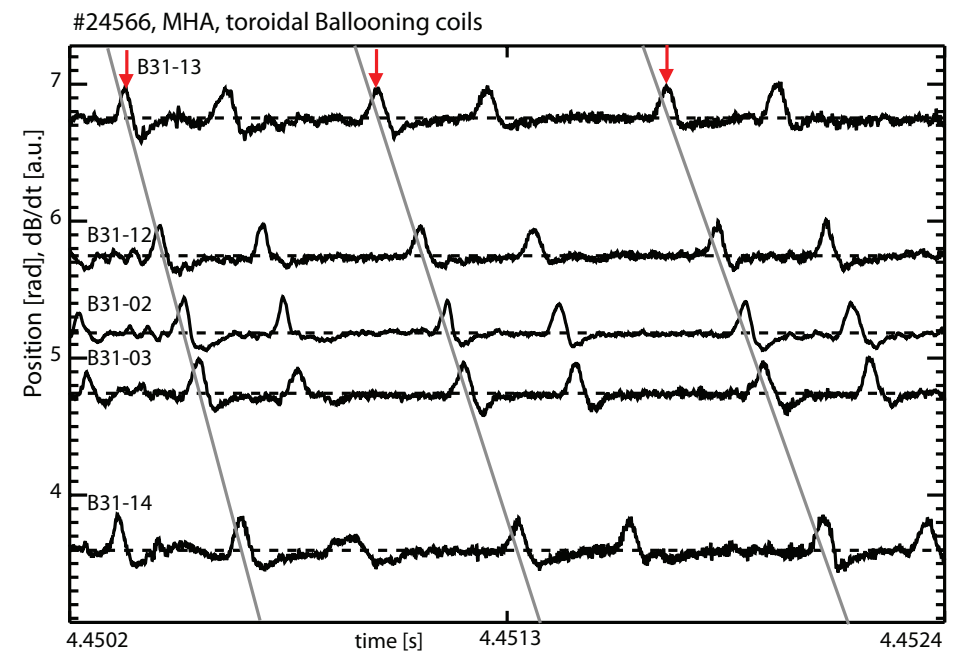

Figure 7. Exotic case of the Edge Snake with an $n=3, m=18$ structure with only 2 measurable current wires.

\section{Discussion}

The experimental characteristics of the Edge Snake are combined and possible theoretical explanations for the drive of this instability are discussed.

\subsection{Simulation of the magnetic time traces}

To ascertain the concept of a poloidally localized current wire, a simulation of the magnetic time traces was performed. The torus is assumed to be an infinite linear structure in the toroidal direction with the actual poloidal cross section of the plasma, determined by equilibrium calculations [7]. The current distribution $j_{\text {pert }} \propto \sin \left(\theta^{*}(\theta) \cdot m\right)^{k} \cdot \delta(r-r(q=6))$ consists of a radial delta function with a poloidal $m=6$ sinusoidal structure on the $q=6$ surface. A high exponent $k$ is used to take into account the strong poloidal localization of the current wire with a FWHM taken from the electron temperature measurement. The current distribution is then corrected for the large dependence of the pitch angle on the poloidal angle $\theta^{*}(\theta)$, taking into account the plasma equilibrium. The vessel, the passive stabilizing loop (PSL) and the divertor including induced currents are ignored in the simulation. The poloidal current distribution and the geometrical orientations are shown in Figure 8.

With these simplifications the magnetic field can be calculated with the formula of Biot Savart. The derivative of the calculated magnetic field for two coils is shown in Figure 9 (a) (dashed red curve) in comparison to the experimental data (solid black curve). The conformity between the simulation and the experiment underlines the existence of a poloidally strongly localized current wire as concluded from the localized 2D ECE measurements of the temperature change. In addition the different shapes of the Mirnov coil signals at different poloidal positions can be reproduced very well (Figure 9 (b)). The distinction of the shapes of the signals at different locations arises from the varying distance and orientation of the coils to 

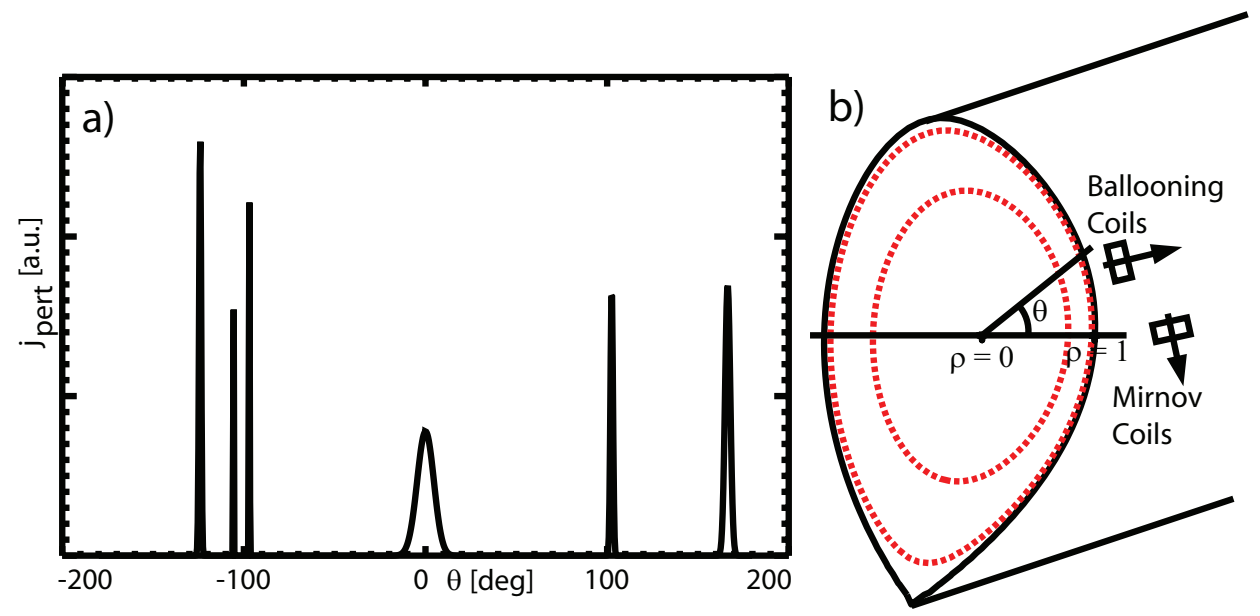

Figure 8. (a) Poloidal current distribution used for the simulation, corrected for the large dependence of the pitch angle on the poloidal angle $\theta$.

(b) Geometrical setup used for the simulation.

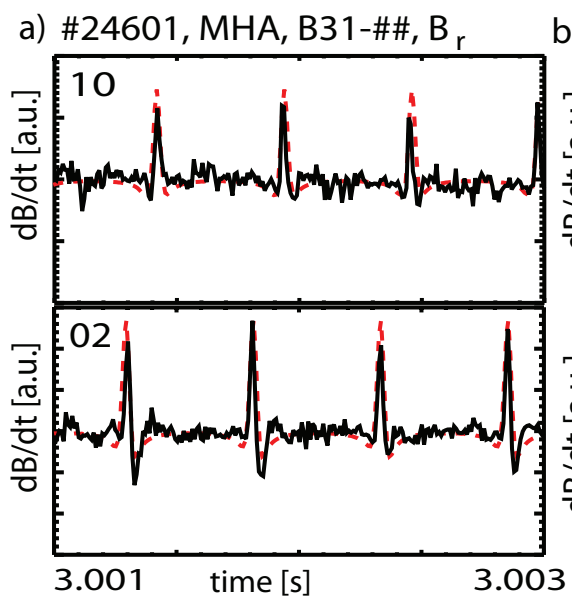

b) \#24601, MHA, C09, -\#\#, $\mathrm{B}_{\text {pol }}$

Figure 9. (a) Simulated time traces of two Ballooning coils (dashed red curve), and (b) 4 Mirnov coils (dashed red curves) showing good accordance with the experimental data (solid black curves) and a good reproduction of the different shapes of the signals.

the plasma. Thus the magnetic signals including their different shapes can be explained by a poloidally very localized defect current wire.

\subsection{Possible explanation as a magnetic island with a neoclassical drive}

Most of the observed diagnostics show a coherent picture of the Edge Snake. It appears as a magneto-hydro-dynamic instability, which consists of a poloidally very localized current wire aligned with the magnetic field lines and rotates in the electron diamagnetic drift direction. The density as well as the 1D temperature and hence the pressure profiles show a flattening around the $q=6$ surface, which points to a magnetic island with a neoclassical drive as the nature of the Edge Snake. A current density of $0.2 \mathrm{MA} / \mathrm{m}^{2}$ can be calculated flowing against the normal plasma current and annihilating a noticeable part of the unperturbed Bootstrap cur- 
rent at the location of the island. The drive and the long life time of the magnetic island can be explained by this reduction due to the dip in the pressure gradient inside the island, which then has the effect of a further reduced pressure gradient. By reconnection of field lines, a self maintaining island with its own nested magnetic surfaces arises which is similar to the neoclassical tearing mode [12] but in the high gradient zone just inside the separatrix.

Another argument for a magnetic island being the nature of the Edge Snake is the fact that the underlying current on the high field side has to be very localized to explain the measurements observed on the Mirnov coils. A structure unlike a magnetic island, which does not have its own nested magnetic surfaces, but ranges over an area of $q$ values, would be dragged poloidally apart by the radial shear as a result of the strong variation of the connection length of magnetic lines on different magnetic surfaces. This shear stabilization is expected to be especially pronounced in the outer region of the plasma in the steep gradient zone, where the Edge Snake is localized. Consequently it is very likely that the Edge Snake is a magnetic island with its own nested magnetic surfaces.

\subsection{Further remarks}

Owing to the lack of unambiguous experimental data because the measurements are recorded at the temporal, spatial and operational limits of the diagnostics, it is at present not possible to decide on the exact current distribution of the Edge Snake. Hopefully in the campaign 2011 an analysis of the Edge Snake with a 3D magnetic coil on the midplane manipulator on ASDEX Upgrade will clarify this current distribution.

The multiple harmonics and the huge ratio of the poloidal distance to the extension demonstrate that the Edge Snake is not a typical island described in literature so far. It seems to have a poloidally very localized current structure and a large area in between, where the equilibrium is almost unperturbed. Additionally the 2D ECE measurements need some more investigation. Assuming a flattening of the profiles due to a magnetic island two radially adjoining areas are expected, one hotter and more to the outside, one cooler and more to the inside. This deviance from existing theory can be explained by a magnetic island which holds the measured current very localized in the O-point and has a very expanded X-point resembling the quiet phase on the diagnostics. A small tilt of the island could bend the flux surface which would cause the seeming poloidal division of the cooler inner and hotter outer part on the 2D ECE measurement. It is unclear, if such a configuration can survive some tens of milliseconds, but is the most plausible explanation.

A significant change of the confinement was not measured during the investigation. However in some discharges the occurrence of the Edge Snake seems to suppress other magnetohydro-dynamic activities in the plasma, which might be a sign of improved stability of the pedestal. 


\section{Comparison with similar instabilities}

In this section, the Edge Snake will be compared to instabilities with similar characteristics like the Picket Fence Mode [13], the Wash Board Mode [14], the Outer Mode [15, 16] and the Palm Tree Mode [17] at JET and the Edge Harmonic Oscillation [18, 19] at DIII-D and ASDEX Upgrade. All of these modes have in common that they have a fundamental toroidal mode number $n=1$ with many harmonics in the spectrum and similar magnetic, temperature and radiation signals which show an inharmonic structure. They all have a large ratio of poloidal distance to extension and a similar frequency range of several $\mathrm{kHz}$.

\subsection{Picket Fence Mode}

The Picket Fence Mode also rotates in the electron diamagnetic drift direction and is radially strongly localized near the separatrix.

However, in contrast to the Edge Snake, it occurs only in course of a different scenario, namely an L-mode Optimized Shear discharge with an Internal Transport Barrier before the L-H transition and never afterwards. The gradients of electron temperature and density in the area of the instability are much smaller before the transition. Therefore the neoclassical drive is unlikely to be the same for the Edge Snake within the H-mode and the Picket Fence Mode during L-Mode.

\subsection{Wash Board Mode}

Like the Edge Snake, the Wash Board Mode also rotates in the electron diamagnetic drift direction and occurs only during $\mathrm{H}$-mode plasmas.

In contrast it is not strongly radially localized, but seems to extend from the edge further to the inside, sometimes as far as to the centre. Additionally the Wash Board Mode seems to have a strong ballooning but not a tearing mode character, which differentiates it from the Edge Snake.

\subsection{Outer Mode}

Contrary to the Edge Snake the Outer Mode is located well inside the pedestal top, outside the region of steep gradients and is a very robust mode, which even exists during a full Sawtooth cycle. It can be identified as a current driven mode with a current parallel to the plasma current and is likely to be a saturated external kink and not a tearing mode. It is therefore not a candidate for the Edge Snake.

\subsection{Edge Harmonic Oscillation}

On one hand, the Edge Harmonic Oscillation also rotates in the electron diamagnetic drift direction and is localized in the outermost region at a $\rho_{p o l}=0.95-1.0$ with a radial extension 
of only $0.5 \mathrm{~cm}$.

On the other hand, the Edge Harmonic Oscillation has a large enough impact on the particle transport to sustain an ELM free phase, which was not observed during the occurrence of the Edge Snake. Additionally it seems not to be a tearing mode, but a peeling ballooning mode driven by current and the gradients of pressure and rotation at the edge. This also excludes the Edge Harmonic Oscillation as a candidate for the Edge Snake.

\subsection{Palm Tree Mode}

Similar to the Edge Snake the Palm Tree Mode occurs only during type-I ELMy H-modes and has a small radial extent of only a few centimeters. Additionally it is a magnetic island with a defect current.

In contrast to the Edge Snake the Palm Tree Mode is caused by ELMs and is located at the $\mathrm{q}=3$ surface well inside the pedestal top and rotates in the ion diamagnetic drift direction. Its frequency behavior is completely different to the Edge Snake, namely it rises from almost zero to a saturated value of about $3 \mathrm{kHz}$ in contrast to the Edge Snake, which was only observed at a constant frequency. Therefore the Edge Snake can be seen to be different to the Palm Tree Mode.

\section{Conclusion}

The Edge Snake is best modeled as a magnetic island with a neoclassical drive. It originates from a self consistent closed loop of reduction of the electron pressure gradient, which leads to a decrease of the Bootstrap current inside the island which again leads to a reduction of the pressure gradient. The current is poloidally very localized, which becomes manifest in very inharmonic signals with a huge number of harmonics in the spectral range. The Edge Snake has a fundamental toroidal mode number of $\mathrm{n}=1$, a frequency of around $2 \mathrm{kHz}$ and is located in the steep gradient zone in the outermost centimeters of the plasma inside the separatrix. It occurs only during type I ELMy H-modes, but is not clearly related to the ELM cycle. It only relies on the formation of the steep gradient zone during the H-mode. By simulating a poloidally strongly localized current wire it was possible to reproduce the time traces of the magnetic pickup coils.

However, the pattern of the electron temperature change on the 2D ECE measurement and the exact current distribution is not entirely understood yet and requires further investigation.

\section{References}

[1] H. Zohm. Edge localized modes (ELMs). Plasma Phys. Control. Fusion, 38(2):105-128, 1996.

[2] K. Asmussen. Untersuchungen zum Verhalten von Wolfram in Tokamakplasmen. Technical Report 10/2, IPP, Garching, Germany, 1996. PhD thesis TU Munich. 
[3] R.D. Gill, A.W. Edwards, D. Pasini, and W. Weller. Snake-like density perturbations in JET. Nucl. Fusion, 32(5):723-735, 1992.

[4] H.J. Hartfuss, T. Geist, and M. Hirsch. Heterodyne methods in millimetre wave plasma diagnostics with applications to ECE, interferometry and reflectometry. Plasma Phys. Control. Fusion, 39(11):1693, 1997.

[5] B. Reiter, T. Eich, G. Pautasso, C. Fuchs, L. Giannone, M. Maraschek, V. Igochine, A. Flaws, J. Neuhauser, T. Lunt, K. Behler, A. Lohs, G. Schramm, L. Kammerloher, H. Eixenberger, and ASDEX Upgrade Team. Application of AXUV Diodes for broad-band plasma radiation studies in ASDEX Upgrade. 36th EPS Conf. on Plasma Physics. Contributed Papers, 33 E:P-1.161, 2009.

[6] G. Pautasso, L. Giannone, A. Herrmann, M. Bernert, and the ASDEX Upgrade Team. Evolution of the halo current in ASDEX Upgrade disruptions. 37th EPS Conf. on Plasma Physics. Contributed Papers, 33 E:P2.152, 2010.

[7] P.J. McCarthy. Analytical solutions to the Grad-Shafranov equation for tokamak equilibrium with dissimilar source functions. Phys. Plasmas, 6(9):3554-3560, 1999.

[8] M. Schittenhelm. Investigation of Tearing Modes in asymmetric elongated plasmas in the ASDEX Upgrade Tokamak. Technical Report IPP Nr. 1/289, IPP, Garching, Germany, 1995. Ph.D. thesis TU Munich.

[9] R. Fischer, E. Wolfrum, J. Schweizer, and the ASDEX Upgrade Team. Probabilistic lithium beam data analysis. Plasma Phys. Control. Fusion, 50(8):085009 (26pp), 2008.

[10] P.J. McCarthy, L. Horton, A. Kallenbach, B. Kurzan, and ASDEX Upgrade Team. ELM-resolved interpretive MHD equilibria on ASDEX Upgrade using SOL tile currents and kinetic data. 30th EPS Conf. on Plasma Physics. Contributed Papers, 27 A:P-1.64, 2003.

[11] J.E. Boom, I.G.J. Classen, W. Suttrop, N.K. Hicks, E. Wolfrum, B.J. Tobias, H.K. Park, and ASDEX Upgrade Team. 2D ECE-Imaging measurements of Edge Localized Modes (ELMs) at ASDEX Upgrade. 37th EPS Conf. on Plasma Physics. Contributed Papers, 34:P-2.119, 2010.

[12] O. Sauter, R.J. La Haye, Z. Chang, D.A. Gates, Y. Kamada, H. Zohm, A. Bondeson, D. Boucher, J.D. Callen, M.S. Chu, T.A. Gianakon, O. Gruber, R.W. Harvey, C.C. Hegna, L.L. Lao, D.A. Monticello, F. Perkins, A. Pletzer, A.H. Reiman, M. Rosenbluthand E.J. Strait, T.S. Taylor, A.D. Turnbull, F. Waelbroeck, J.C. Wesley, H.R. Wilson, and R. Yoshino. Beta limits in long-pulse tokamak discharges. Phys. Plasmas, 4 (5):1654-1664, 1997.

[13] G.T.A. Huysmans, T.C. Hender, B. Alper, Y.F. Baranov, D. Borba, G.D. Conway, G.A. Cottrell, C. Gormezano, P. Helander, O.J. Kwon, M.F.F. Nave, A.C.C. Sips, F.X. Söldner, E.J. Strait, W.P. Zwingmann, and JET Team. MHD stability of optimized shear discharges in JET. Nucl. Fusion, 39(11):1489-1507, 1999.

[14] P. Smeulders, G.D. Conway, B. Alper, B. Balet, D.V. Bartlett, D. Borba, N. Deliyanakis, T.C. Hender, and O.J. Kwon. Characteristics of a new Class of Transport related MHD modes in JET H-mode Plasmas. Plasma Phys. Control. Fusion, 41(10):1303-1320, 1999.

[15] E.R. Solano, P.J. Lomas, B. Alper, G.S. Xu, Y. Andrew, G. Arnoux, A. Boboc, L. Barrera, P. Belo, M.N.A. Beurskens, M. Brix, K. Crombe, E. de la Luna, S. Devaux, T. Eich, S. Gerasimov, C. Giroud, D. Harting, D. Howell, A. Huber, G. Kocsis, A. Korotkov, A. Lopez-Fraguas, M.F.F. Nave, E. Rachlew, F. Rimini, S. Saarelma, A. Sirinelli, S.D. Pinches, H. Thomsen, L. Zabeo, and D. Zarzoso. Observation of Confined Current Ribbon in JET Plasmas. Phys. Rev. Letters, 104(18):185003, May 2010.

[16] G.T.A. Huysmans, T.C. Hender, and B. Alper. Identification of external kink modes in JET. Nucl. Fusion, 38(2):179-187, 1998.

[17] H.R. Koslowski, B. Alper, D.N. Borba, T. Eich, S.E. Sharapov, C.P. Perez, E. Westerhof, and JET-EFDA contributors. Observation of the palm tree mode, a new MHD mode excited by type-I ELMs on JET. Nucl. Fusion, 45(3):201-208, 2005.

[18] K.H. Burrell, T.H. Osborne, P.B. Snyder, W.P. West, M.E. Fenstermacher, R.J. Groebner, P. Gohil, A.W. Leonard, and W.M. Solomon. Edge pedestal control in quiescent H-mode discharges in DIII-D using co-plus counter-neutral beam injection. Nucl. Fusion, 49(8):085024 (9pp), 2009.

[19] W. Suttrop, M. Maraschek, G.D. Conway, H.U. Fahrbach, G. Haas, L.D. Horton, T. Kurki-Suonio, C.J. Lasnier, A.W. Leonard, C.F. Maggi, H. Meister, A. Mück, R. Neu, I. Nunes, Th. Pütterich, M. Reich, 
A.C.C. Sips, and the ASDEX Upgrade Team. ELM-free stationary H-mode plasmas in the ASDEX Upgrade tokamak. Plasma Phys. Control. Fusion, 45(8):1399-1416, 2003. 